\title{
Guest Editorial: Special Section on Multimedia Semantics
}

\author{
B. Prabhakaran
}

Published online: 2 December 2009

(C) 2009 Springer Science+Business Media, LLC. Manufactured in The United States

Multimedia semantics is the research area that deals with interpreting the meaning of a multimedia content and annotate the data in such a way that it will be useful for others to search, reuse, and repurpose the content (assuming the appropriate copyrights are available to do them!). The major hurdle in achieving this objective is well known: the binary nature of multimedia content does not lend itself to precise interpretations easily. Several machine learning and other artificial intelligence techniques have been developed for analyzing the binary data. However, these approaches have two major deficiencies in general:

- While these approaches are able to recognize low level features, they suffer from low accuracies in identifying high level concepts that requires combinations of low level features, e.g., gestures in video or a location in an image.

- In many cases, there is lot of ambiguity in recognizing low level features also. These ambiguities arise because of the probabilistic nature of recognition on noisy input data.

In many cases, the above deficiencies can be overcome by using knowledge or information from another media. For instance, ambiguities in low level feature recognition can be resolved to a great extent by considering "context" among the recognized features. As an example, consider an image where the background has been identified as "desert". It may be highly unlikely a marine living thing can be associated with this context of desert. Hence, a lower probability can be assigned to this recognition of the feature. Several techniques have been suggested to facilitate the understanding and usage of the context in order to improve the accuracy of annotating the multimedia content.

B. Prabhakaran $(\bowtie)$

University of Texas at Dallas,

Dallas, TX 75080, USA

e-mail: bprabhakaran@utdallas.edu
This special section on multimedia semantics is devoted to research works that harbor on these techniques. Earlier version of some of these researches were presented in a special session associated with the IEEE International Conference on Semantic Computing 2007 (ICSC'07). The first paper in this special section is by Hauptmann et al. on "A MultiPronged Approach to Improving Semantic Extraction of News Video". Here, the authors present a discriminative learning framework called Multi-concept Discriminative Random Field (MDRF) that combines low level features and high level concepts for annotating news video. Authors use TRECVID data set to experimentally also show that no single approach is able to provide better accuracy for concept detection, and hence recommend the use of multiple resources to achieve significant results.

The next paper is by Yohan Jin et al. on the topic 'Knowledge Based Image Annotation Refinement'. Here, the authors use distance between keywords based on WordNet as a knowledge base for resolving ambiguities in image annotations. Building on this approach, they formulate the image annotation problem as a graph partitioning problem. Considering the fact that the possibilities for annotations can be large, they propose a randomized graph partitioning algorithm after proving that the problem is NP-hard. They use benchmark image data set to validate their approach.

The last paper is by J-W. Kim et al. on "Organization and Tagging of Blog and News Entries based on Content Reuse". This paper focuses on the relatively new but popular medium of communication - blogs. The first problem encountered is one that of understanding the relationships among the numerous blogs in the "blogosphere". For this, it leverages on the fact that related blogs contain similar quotes and commentaries depending on the topic. Based on the "flow" of these quotes, it organizes these blogs. Next, it considers the quotes as contexts that can lead to effective tagging of the blog content.

In conclusion, this special section gives a quick, quality snap shot of this important field of research: multimedia 
semantics. We believe that the papers in this special section will stimulate further research leading to better techniques for understanding multimedia content.

Acknowledgement We would like to thank all authors for their contributions and patience with the rounds of changes required as suggested by reviewers. We are grateful to all the reviewers who did an excellent job by providing detailed and useful comments on the papers.

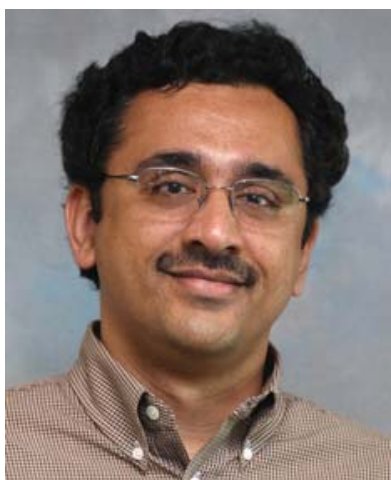

Dr. B. Prabhakaran is an Associate Professor with the faculty of Computer Science Department, University of Texas at Dallas. He has been working in the area of multimedia systems : animation \& multimedia databases, authoring \& presentation, resource management, and scalable web-based multimedia presentation servers. Dr. Prabhakaran received the prestigious National Science Foundation (NSF) CAREER Award in 2003 for his proposal on Animation Databases. He is also the Principal Investigator for US Army Research Office (ARO) grant on 3D data storage, retrieval, and delivery. He has published several research papers in various refereed conferences and journals in this area.

He has served as an Associate Chair of the ACM Multimedia Conferences in 2006 (Santa Barbara), 2003 (Berekeley, CA), 2000 (Los Angeles, CA) and in 1999 (Orlando, FL) He has served as guest-editor of special issues on various topics for different multimedia journals. He is also serving on the editorial board of journals such as Multimedia Systems (Springer), Multimedia Tools and Applications (Springer), Journal of Multimedia (Academy Publishers), and Journal of Multimedia Data Engineering and Management (Information Resources Management Association (IRMA)). He is also the Editor-in-chief of the ACM SIGMM (Special Interest Group on Multimedia) Online magazine. He has also served as program committee member on several multimedia conferences and workshops. He has presented tutorials in ACM Multimedia and other multimedia conferences.

Dr Prabhakaran has served as a visiting research faculty with the Department of Computer Science, University of Maryland, College Park. He also served as a faculty in the Department of Computer Science, National University of Singapore as well as in the Indian Institute of Technology, Madras, India. 